\title{
On the modified discrete KP equation with self-consistent sources
}

Gegenhasi, Xiaorong Bai

To cite this article: Gegenhasi, Xiaorong Bai (2017) On the modified discrete KP equation with self-consistent sources, Journal of Nonlinear Mathematical Physics 24:2, 224-238, DOI: https://doi.org/10.1080/14029251.2017.1313476

To link to this article: https://doi.org/10.1080/14029251.2017.1313476

Published online: 04 January 2021 


\title{
On the modified discrete KP equation with self-consistent sources
}

\author{
Gegenhasi \\ School of Mathematical Science, Inner Mongolia University, No.235 West College Road \\ Hohhot, Inner Mongolia 010021, P.R. China \\ gegenhasiimu@126.com \\ Xiaorong Bai \\ School of Mathematical Science, Inner Mongolia University, No.235 West College Road \\ Hohhot, Inner Mongolia 010021, P.R. China \\ 1587324571@qq.com
}

Received 25 July 2016

Accepted 13 March 2017

\begin{abstract}
The modified discrete KP equation is the Bäcklund transformation for the Hirota's discrete KP equation or the Hirota-Miwa equation. We construct the modified discrete KP equation with self-consistent sources via source generation procedure and clarify the algebraic structure of the resulting coupled modified discrete KP system by presenting its discrete Gram-type determinant solutions. It is also shown that the commutativity between the source generation procedure and Bäcklund transformation is valid for the discrete KP equation. Finally, we demonstrate that the modified discrete KP equation with self-consistent sources yields the modified differential-difference KP equation with self-consistent sources through a continuum limit. The continuum limit of an explicit solution to the modified discrete KP equation with self-consistent sources also gives the explicit solution for the modified differential-difference KP equation with self-consistent sources.
\end{abstract}

Keywords: Modified discrete KP equation, Source generalization procedure, Discrete Gram-type determinant, Continuum limit

2000 Mathematics Subject Classification: 37K10, 37K40

\section{Introduction}

The study of discrete integrable systems has attracted a lot of interest in recent years. The most important and widely studied three-dimensional discrete integrable system is the Hirota-Miwa equation (or discrete KP equation in bilinear form ) [1]- [3]:

$$
\begin{aligned}
& a_{1}\left(a_{2}-a_{3}\right) f\left(k_{1}+a_{1}\right) f\left(k_{2}+a_{2}, k_{3}+a_{3}\right)+a_{2}\left(a_{3}-a_{1}\right) f\left(k_{2}+a_{2}\right) f\left(k_{1}+a_{1}, k_{3}+a_{3}\right) \\
& +a_{3}\left(a_{1}-a_{2}\right) f\left(k_{3}+a_{3}\right) f\left(k_{1}+a_{1}, k_{2}+a_{2}\right)=0
\end{aligned}
$$

where $f=f\left(k_{1}, k_{2}, k_{3}\right)$ and $a_{1}, a_{2}, a_{3}$ are the difference intervals for discrete independent variables $k_{1}, k_{2}, k_{3}$, respectively. We omit the dependence on unshifted variables in the above equation and in the following notations. The Hirota-Miwa equation (1.1) is considered as a master equation which by performing scaling continuum limit gives many well-known semi-discrete and continuous integrable systems. 
Under the variable transformations $k_{1} \rightarrow a_{1} k_{1}, k_{2} \rightarrow a_{2} k_{2}, k_{3} \rightarrow a_{3} k_{3}$, the discrete KP equation (1.1) can be written in a compact form by using bilinear operator:

$$
\left[a_{1}\left(a_{2}-a_{3}\right) e^{\frac{1}{2} D_{k_{1}}-\frac{1}{2} D_{k_{2}}-\frac{1}{2} D_{k_{3}}}+a_{2}\left(a_{3}-a_{1}\right) e^{\frac{1}{2} D_{k_{2}}-\frac{1}{2} D_{k_{1}}-\frac{1}{2} D_{k_{3}}}+a_{3}\left(a_{1}-a_{2}\right) e^{\frac{1}{2} D_{k_{3}}-\frac{1}{2} D_{k_{1}}-\frac{1}{2} D_{k_{2}}}\right] f \cdot f=0,
$$

where the bilinear operator is defined by

$$
e^{\delta D_{n}} a(n) \cdot b(n)=a(n+\delta) b(n-\delta) .
$$

The discrete KP equation (1.1) or (1.2) has the following Bäcklund transformation [1,4]:

$$
\begin{aligned}
& {\left[a_{1} e^{\frac{1}{2} D_{k_{1}}-\frac{1}{2} D_{k_{2}}}-a_{2} e^{\frac{1}{2} D_{k_{2}}-\frac{1}{2} D_{k_{1}}}+\left(a_{2}-a_{3}\right) e^{\frac{1}{2} D_{k_{1}}+\frac{1}{2} D_{k_{2}}}\right] f \cdot g=0} \\
& {\left[a_{1} e^{\frac{1}{2} D_{k_{1}}-\frac{1}{2} D_{k_{3}}}-a_{1} e^{\frac{1}{2} D_{k_{3}}-\frac{1}{2} D_{k_{1}}}+\left(a_{3}-a_{1}\right) e^{\frac{1}{2} D_{k_{1}}+\frac{1}{2} D_{k_{3}}}\right] f \cdot g=0 .}
\end{aligned}
$$

Through the variable transformations $k_{1} \rightarrow \frac{1}{a_{1}} k_{1}, k_{2} \rightarrow \frac{1}{a_{2}} k_{2}, k_{3} \rightarrow \frac{1}{a_{3}} k_{3}$, the modified discrete KP equations (1.4-1.5) become

$$
\begin{aligned}
& a_{1} f\left(k_{1}+a_{1}\right) g\left(k_{2}+a_{2}\right)-a_{2} f\left(k_{2}+a_{2}\right) g\left(k_{1}+a_{1}\right)+\left(a_{2}-a_{1}\right) f\left(k_{1}+a_{1}, k_{2}+a_{2}\right) g=0 \\
& a_{1} f\left(k_{1}+a_{1}\right) g\left(k_{3}+a_{3}\right)-a_{3} f\left(k_{3}+a_{3}\right) g\left(k_{1}+a_{1}\right)+\left(a_{3}-a_{1}\right) f\left(k_{1}+a_{1}, k_{3}+a_{3}\right) g=0 .
\end{aligned}
$$

Soliton equations with self-consistent sources, describing important physical processes, have been studied by means of various mathematical approachs such as inverse scattering methods [5][9], Darboux transformation methods [10]- [13], Hirota's bilinear method and Wronskian technique [14]- [22]. However, most results have been achieved in continuous case. Comparatively less work has been done in discrete case, especially in the fully discrete case.

In [23], the authors propose a new algebraic method, called the source generalization procedure, to construct and solve the soliton equations with self consistent sources both in continuous and discrete cases. The purpose of this paper is to utilize the source generalization procedure to the modified discreet KP equation (1.6-1.7) to construct the modified discrete KP equation with selfconsistent sources and clarify the bilinear structures of the modified discrete KP equations with self-consistent sources. Furthermore, we verity that the commutativity of the source generation procedure and Bäcklund transformation holds for the fully discrete case.

The outline of this paper is as follows. In Sec.2, we construct the modified discrete KP equations with self-consistent sources and give their discrete Gram-type determinant solutions. Sec. 3 is devoted to show that the commutativity of the source generation procedure and Bäcklund transformation is valid for the discrete KP equation. We end this paper with a summary and discussion in section 4.

\section{The modified discrete KP equation with self-consistent sources}

In this section, we construct the modified discrete KP equation with self-consistent sources via source generation procedure. Furthermore, we present the discrete Gram-type determinant solution for the modified discrete KP equation with self-consistent sources and clarify its bilinear structure. 
It is given in [24] that the bilinear difference equations (1.6-1.7) have the following discrete Gram-type determinant solution:

$$
\begin{gathered}
f\left(k_{1}, k_{2}, k_{3}\right)=|M|, \\
g\left(k_{1}, k_{2}, k_{3}\right)=\left|\begin{array}{cc}
M & \Phi(-1) \\
\bar{\Phi}(0)^{T} & 1
\end{array}\right| .
\end{gathered}
$$

where $M=\operatorname{det}\left(c_{i j}+m_{i j}\right)_{1 \leq i, j \leq N}$ in which $c_{i j}(1 \leq i, j \leq N)$ is arbitrary constant and the matrix element $m_{i j}(1 \leq i, j \leq N)$ is an function of $k_{1}, k_{2}, k_{3}$ satisfying the difference equation

$$
\begin{aligned}
& \Delta_{+k_{v}} m_{i j}=\phi_{i}\left(k_{v}+a_{v} ; 0\right) \bar{\phi}_{j}(0), \\
& i, j=1,2, \cdots, N, v=1,2,3,
\end{aligned}
$$

where unshifted independent variables are suppressed and $\phi i$ and $\bar{\phi}_{i}$ are arbitrary functions of $k_{1}, k_{2}, k_{3}$ and an integer $\mathrm{s}$, satisfying the dispersion relations

$$
\begin{aligned}
& \Delta_{-k_{v}} \phi_{i}\left(k_{1}, k_{2}, k_{3}, s\right)=\phi_{i}\left(k_{1}, k_{2}, k_{3}, s+1\right), \\
& \Delta_{+k_{v}} \bar{\phi}_{i}\left(k_{1}, k_{2}, k_{3}, s\right)=\bar{\phi}_{i}\left(k_{1}, k_{2}, k_{3}, s+1\right),
\end{aligned}
$$

where $\Delta_{-k_{v}}, \Delta_{+k_{v}}$ are defined by

$$
\begin{aligned}
& \Delta_{-k_{v}} F\left(k_{v}\right)=\frac{F\left(k_{v}\right)-F\left(k_{v}-a_{v}\right)}{a_{v}}, \\
& \Delta_{+k_{v}} F\left(k_{v}\right)=\frac{F\left(k_{v}+a_{v}\right)-F\left(k_{v}\right)}{a_{v}}, v=0,1,2,3 .
\end{aligned}
$$

In addition, $\Phi(s), \bar{\Phi}(s)$ (s is an integer) are $N$ th column vectors defined by

$$
\begin{aligned}
& \Phi(s)=\left(\phi_{1}(s), \phi_{2}(s), \cdots, \phi_{N}(s)\right)^{T}, \\
& \bar{\Phi}(s)=\left(\bar{\phi}_{1}(s), \bar{\phi}_{2}(s), \cdots, \bar{\phi}_{N}(s)\right)^{T} .
\end{aligned}
$$

In order to construct the modified fully discrete KP equation with self-consistent sources, we change the discrete Gram-type determinant solutions (2.1-2.2) into the following form:

$$
\begin{gathered}
f\left(k_{1}, k_{2}, k_{3}\right)=\operatorname{det}\left(c_{i j}\left(k_{1}\right)+m_{i j}\right)_{1 \leq i, j \leq N}=|D|, \\
g\left(k_{1}, k_{2}, k_{3}\right)=\left|\begin{array}{cc}
D & \Phi(-1) \\
\bar{\Phi}(0)^{T} & 1
\end{array}\right|,
\end{gathered}
$$

where $N \times N$ matrix $D=\left(c_{i j}\left(k_{1}\right)+m_{i j}\right)_{1 \leq i, j \leq N}$ and $m_{i j}, \Phi(s), \bar{\Phi}(s)$ are given in (2.3-2.7). In addition, $c_{i j}\left(k_{1}\right)$ satisfies

$$
c_{i j}\left(k_{1}\right)=\left\{\begin{array}{lc}
c_{i}\left(k_{1}\right), & 1 \leq i \leq K \leq N \text { and } j=1,2, \cdots, K, K \in Z^{+}, \\
d_{i j}, & \text { otherwise },
\end{array}\right.
$$

with $c_{i}\left(k_{1}\right)$ being an arbitrary function of $k_{1}$ and $K$ being a positive integer. Then we have the following difference formula by employing Eqs. (2.1-2.5): 


$$
\begin{aligned}
& f\left(k_{1}, k_{2}, k_{3}\right)=\left|\begin{array}{cc}
M_{d} C\left(k_{1}\right) \\
\alpha^{T} & 1
\end{array}\right|, \quad g\left(k_{1}, k_{2}, k_{3}\right)=\left|\begin{array}{ccc}
M_{d} & C\left(k_{1}\right) \Phi(-1) \\
a^{T} & 1 & 0 \\
\bar{\Phi}(0)^{T} & 0 & 1
\end{array}\right| \\
& f\left(k_{\mu}+a_{\mu}\right)=\alpha_{\mu}\left|\begin{array}{ccc}
M_{d} & C\left(k_{1}\right) \Phi\left(0_{\mu}\right) \\
\alpha^{T} & 1 & 0 \\
-\bar{\Phi}(0)^{T} & 0 & a_{\mu}^{-1}
\end{array}\right|, \quad f\left(k_{1}+a_{1}\right)=a_{1}\left|\begin{array}{ccc}
M_{d} & C\left(k_{1}+a_{1}\right) \Phi\left(0_{1}\right) \\
\alpha^{T} & 1 & 0 \\
-\bar{\Phi}(0)^{T} & 0 & a_{1}^{-1}
\end{array}\right|, \\
& f\left(k_{2}+a_{2}, k_{3}+a_{3}\right)=\frac{\left(a_{2} a_{3}\right)^{2}}{\left(a_{2}-a_{3}\right)}\left|\begin{array}{cccc}
M_{d} & C\left(k_{1}\right) & \Phi\left(0_{2}\right) & \Phi\left(0_{3}\right) \\
\alpha^{T} & 1 & 0 & 0 \\
-\bar{\Phi}(1)^{T} & 0 & -a_{2}^{-2} & -a_{3}^{-2} \\
-\bar{\Phi}(0)^{T} & 0 & a_{2}^{-1} & a_{3}^{-1}
\end{array}\right| \\
& f\left(k_{1}+a_{1}, k_{\mu}+a_{\mu}\right)=\frac{\left(a_{1} a_{\mu}\right)^{2}}{\left(a_{1}-a_{\mu}\right)}\left|\begin{array}{cccc}
M_{d} & C\left(k_{1}+a_{1}\right) & \Phi\left(0_{1}\right) & \Phi\left(0_{\mu}\right) \\
\alpha^{T} & 1 & 0 & 0 \\
-\bar{\Phi}(1)^{T} & 0 & -a_{1}^{-2} & -a_{\mu}^{-2} \\
-\bar{\Phi}(0)^{T} & 0 & a_{1}^{-1} & a_{\mu}^{-1}
\end{array}\right| \\
& g\left(k_{\mu}+a_{\mu}\right)=-a_{\mu}^{2}\left|\begin{array}{cccc}
M_{d} & C\left(k_{1}\right) \Phi(-1) & \Phi\left(0_{\mu}\right) \\
\alpha^{T} & 1 & 0 & 0 \\
\bar{\Phi}(1)^{T} & 0 & 0 & a_{\mu}^{-2} \\
\bar{\Phi}(0)^{T} & 0 & 1 & -a_{\mu}^{-1}
\end{array}\right|, g\left(k_{1}+a_{1}\right)=-a_{1}^{2}\left|\begin{array}{cccc}
M_{d} & C\left(k_{1}+a_{1}\right) \Phi(-1) \Phi\left(0_{1}\right) \\
\alpha^{T} & 1 & 0 & 0 \\
\bar{\Phi}(1)^{T} & 0 & 0 & a_{1}^{-2} \\
\bar{\Phi}(0)^{T} & 0 & 1 & -a_{1}^{-1}
\end{array}\right|
\end{aligned}
$$

where $\mu=2,3 ; C\left(k_{1}\right), \alpha, \Phi\left(s_{V}\right)$ are $N$ th column vectors given by

$$
\begin{aligned}
& C\left(k_{1}\right)=\left(c_{1}\left(k_{1}\right), \cdots, c_{K}\left(k_{1}\right), 0, \cdots, 0\right)^{T}, \\
& \alpha=(-1, \cdots,-1,0, \cdots, 0)^{T}, \quad \text { number of }-1=K, \\
& \Phi\left(s_{v}\right)=\left(\phi_{1}\left(k_{v}+a_{v} ; s\right), \phi_{2}\left(k_{v}+a_{v} ; s\right) \cdots, \phi_{N}\left(k_{v}+a_{v} ; s\right) ;\right)^{T}, v=1,2,3,
\end{aligned}
$$

and $M_{d}$ is given by

$$
M_{d}=\left|\begin{array}{cccccc}
m_{11} & \cdots & m_{1 k} & m_{1, k+1}+d_{1, k+1} & \cdots & m_{1 N}+d_{1 N} \\
\vdots & \vdots & \vdots & \vdots & \vdots & \vdots \\
m_{k 1} & \cdots & m_{k k} & m_{k, k+1}+d_{k, k+1} & \cdots & m_{k N}+d_{k N} \\
m_{k+1,1}+d_{k+1,1} & \cdots & m_{k+1, k}+d_{k+1, k} & m_{k+1, k+1}+d_{k+1, k+1} & \cdots & m_{k+1, N}+d_{k+1, N} \\
\vdots & \vdots & \vdots & \vdots & \vdots & \vdots \\
m_{N 1}+d_{N 1} & \cdots & m_{N k}+d_{N k} & m_{N, k+1}+d_{N, k+1} & \cdots & m_{N N}+d_{N N}
\end{array}\right| .
$$


According to the above results, $f, g$ defined in (2.1-2.2) will not satisfy the modified fully discrete KP equation (1.6-1.7) again. In fact, they satisfy the following equations:

$$
\begin{gathered}
a_{1} f\left(k_{1}+a_{1}\right) g\left(k_{2}+a_{2}\right)-a_{2} f\left(k_{2}+a_{2}\right) g\left(k_{1}+a_{1}\right)+\left(a_{2}-a_{1}\right) f\left(k_{1}+a_{1}, k_{2}+a_{2}\right) g\left(k_{1}, k_{2}, k_{3}\right) \\
=a_{2} \sum_{i, j=1}^{K} P_{i, j}\left(k_{1}+a_{1}\right) Q_{i, j}\left(k_{2}+a_{2}\right) \\
a_{1} f\left(k_{1}+a_{1}\right) g\left(k_{3}+a_{3}\right)-a_{3} f\left(k_{3}+a_{3}\right) g\left(k_{1}+a_{1}\right)+\left(a_{3}-a_{1}\right) f\left(k_{1}+a_{1}, k_{3}+a_{3}\right) g\left(k_{1}, k_{2}, k_{3},\right) \\
=a_{3} \sum_{i, j=1}^{K} P_{i, j}\left(k_{1}+a_{1}\right) Q_{i, j}\left(k_{3}+a_{3}\right)
\end{gathered}
$$

where $P_{i, j}, Q_{i, j}$ for $r, m=1, \cdots, K$ are functions of variables $k_{1}, k_{2}, k_{3}$ given by

$$
\begin{gathered}
P_{i, j}\left(k_{1}, k_{2}, k_{3}\right)=\sqrt{c_{i}\left(k_{1}\right)-c_{i}\left(k_{1}-a_{1}\right)}\left|\begin{array}{cc}
D_{i j} & R_{i j} \\
\bar{\Phi}_{j}(0)^{T} & \bar{\phi}_{j}(0)
\end{array}\right|, \\
Q_{i, j}\left(k_{1}, k_{2}, k_{3}\right)=-\sqrt{\Delta c_{i}\left(k_{1}\right)}\left|\begin{array}{cc}
D_{i j} & \Phi_{i}(-1) \\
M_{i j}^{T} & \phi_{i}(-1)
\end{array}\right|,
\end{gathered}
$$

in which $D_{i j}$ is the $(N-1)$ th order matrice obtained by eliminating the $i$ th row and the $j$ th column from the matrice $\left(c_{i, j}\left(k_{2}\right)+m_{i j}\left(k_{1}, k_{2}, k_{3}\right)\right)_{1 \leq i, j \leq N} ; \Phi_{j}(s), \bar{\Phi}_{j}(s)$ are $(N-1)$ th column vectors obtained by eliminating the jth element from $\Phi(s)$ and $\bar{\Phi}(s)$, respectively; $\Delta c_{i}\left(k_{1}\right)$ is defined by

$$
\Delta c_{i}\left(k_{1}\right)=c_{i}\left(k_{1}+a_{1}\right)-c_{i}\left(k_{1}\right)
$$

and $R_{i j}, M_{i j}$ are given by

$$
\begin{gathered}
R_{i j}=\left(c_{1}\left(k_{1}\right)+m_{1 j}, \cdots, c_{i-1}\left(k_{1}\right)+m_{i-1, j}, c_{i+1}\left(k_{1}\right)+m_{i+1, j}, \cdots,\right. \\
\left.c_{K}\left(k_{1}\right)+m_{K, j}, d_{K+1, j}+m_{K+1, j}, \cdots, d_{N, j}+m_{N, j}\right)^{T}, \\
M_{i j}=\left(c_{i}\left(k_{1}\right)+m_{i 1}, \cdots, c_{i}\left(k_{1}\right)+m_{i, j-1}, c_{i}\left(k_{1}\right)+m_{i, j+1}, \cdots,\right. \\
\left.c_{i}\left(k_{1}\right)+m_{i, K}, d_{i, K+1}+m_{i, K+1}, \cdots, d_{i, N}+m_{i, N}\right)^{T}
\end{gathered}
$$

We can also show that functions (2.1-2.2) and new fields (2.19-2.20) for $r, m=1, \cdots, K$ satisfy the following bilinear equations:

$$
\begin{gathered}
a_{2} f\left(k_{2}+a_{2}\right) P_{i j}\left(k_{3}+a_{3}\right)-a_{3} f\left(k_{3}+a_{3}\right) P_{i j}\left(k_{2}+a_{2}\right)+ \\
\left(a_{3}-a_{2}\right) f\left(k_{2}+a_{2}, k_{3}+a_{3}\right) P_{i j}\left(k_{1}, k_{2}, k_{3}\right)=0, \\
a_{2} Q_{i j}\left(k_{2}+a_{2}\right) g\left(k_{3}+a_{3}\right)-a_{3} Q_{i j}\left(k_{3}+a_{3}\right) g\left(k_{2}+a_{2}\right)+ \\
\left(a_{3}-a_{2}\right) Q_{i j}\left(k_{2}+a_{2}, k_{3}+a_{3}\right) g\left(k_{1}, k_{2}, k_{3}\right)=0 .
\end{gathered}
$$

So equations (2.17-2.18,2.22-2.23) constitute the modified discrete KP equation with selfconsistent sources. In the following, we will verify that functions (2.1-2.2) and (2.19-2.20) for $r, m=1, \cdots, K$ solve bilinear equations (2.17-2.18,2.22-2.23).

We can compute the following difference formula for $P_{i, j}, Q_{i, j}, r, m=1, \cdots, K$ by employing equations (2.3-2.5): 


$$
\begin{aligned}
& P_{i j}\left(k_{1}+a_{1}\right)=a_{1}^{2} \sqrt{\Delta C_{i}\left(k_{1}\right)}\left|\begin{array}{ccc}
E_{i j} & \Phi_{i}\left(0_{1}\right) & N_{i j} \\
-\bar{\Phi}_{j}(1)^{T} & -a_{1}^{-2} & -\bar{\phi}_{j}(1)^{T} \\
-\bar{\Phi}_{j}(0)^{T} & a_{1}^{-1} & -\bar{\phi}_{j}(0)^{T}
\end{array}\right| \\
& P_{i, j}\left(k_{v}+a_{v}\right)=-a_{v}^{2} \sqrt{c_{i}\left(k_{1}\right)-c_{i}\left(k_{1}-a_{1}\right)}\left|\begin{array}{ccc}
D_{i j} & R_{i j} & \Phi_{i}\left(0_{v}\right) \\
-\bar{\Phi}_{j}(1)^{T} & -\bar{\phi}_{j}(1) & -a_{v}^{-2} \\
-\bar{\Phi}_{j}(0)^{T} & -\bar{\phi}_{j}(0) & a_{v}^{-1}
\end{array}\right|, \\
& Q_{i, j}\left(k_{v}+a_{v}\right)=a_{v} \sqrt{\Delta c_{i}\left(k_{1}\right)}\left|\begin{array}{ccc}
D_{i j} & \Psi_{i}(-1) & \Psi_{i}\left(0_{v}\right) \\
-\Phi_{j}(0)^{T} & -1 & a_{v}^{-1} \\
M_{i j}^{T} & \varphi_{i}(-1) & \varphi_{i}\left(0_{v}\right)
\end{array}\right|, \\
& Q_{i, j}\left(k_{2}+a_{2}, k_{3}+a_{3}\right)=\frac{a_{2}^{2} a_{3}^{2}}{a_{2}-a_{3}} \sqrt{\Delta c_{i}\left(k_{1}\right)}\left|\begin{array}{cccc}
D_{i j} & \Phi_{i}(-1) & \Phi_{i}\left(0_{2}\right) & \Phi_{i}\left(0_{3}\right) \\
-\bar{\Phi}_{j}(1)^{T} & 0 & -a_{2}^{-2} & -a_{3}^{-2} \\
M_{i j}^{T} & \phi_{i}(-1) & \phi_{i}\left(0_{2}\right) & \phi_{i}\left(0_{3}\right) \\
-\bar{\Phi}_{j}(0)^{T} & -1 & a_{2}^{-1} & a_{3}^{-1}
\end{array}\right|,
\end{aligned}
$$

where $v=2,3, E_{i j}$ is the $(N-1)$ th order matrice obtained by eliminating the $i$ th row and the $j$ th column from the matrix $\left(c_{i, j}\left(k_{1}+a_{1}\right)+m_{i j}\left(k_{1}, k_{2}, k_{3}\right)\right)_{1 \leq i, j \leq N}$, respectively; $\Phi_{i}\left(s_{V}\right)$ is $(N-1)$ th column vector obtained by eliminating the $j$ th element from $\Phi\left(s_{v}\right)$. In addition, $N_{i j}$ is given by

$$
\begin{gathered}
N_{i j}=\left(c_{1}\left(k_{1}+a_{1}\right)+m_{i j}, \cdots, c_{i-1}\left(k_{1}+a_{1}\right)+m_{i-1, j}, c_{i+1}\left(k_{1}+a_{1}\right)+m_{i+1, j}, \cdots,\right. \\
\left.c_{K}\left(k_{1}+a_{1}\right)+m_{K, j}, d_{K+1, j}+m_{K+1, j}, \cdots, d_{N, j}+m_{N, j}\right)^{T} .
\end{gathered}
$$

Substituting equations (2.11-2.15) and (2.24-2.27) into modified discrete KP equation (1.6), we obtain the following determinant identity:

$$
\begin{aligned}
& -\left|\begin{array}{ccc}
M_{d} & C\left(k_{1}+a_{1}\right) & \Phi\left(0_{1}\right) \\
a^{T} & 1 & 0 \\
-\bar{\Phi}(0)^{T} & 0 & a_{1}^{-1}
\end{array}\right|\left|\begin{array}{cccc}
M_{d} & C\left(k_{1}\right) & \Phi(-1) & \Phi\left(0_{2}\right) \\
a^{T} & 1 & 0 & 0 \\
-\bar{\Phi}(1)^{T} & 0 & 0 & -a_{2}^{-2} \\
-\bar{\Phi}(0)^{T} & 0 & -1 & a_{2}^{-1}
\end{array}\right| \\
& +\left|\begin{array}{ccc}
M_{d} & C\left(k_{1}\right) & \Phi\left(0_{2}\right) \\
a^{T} & 1 & 0 \\
-\bar{\Phi}(0)^{T} & 0 & a_{2}^{-1}
\end{array}\right|\left|\begin{array}{cccc}
M_{d} & C\left(k_{1}+a_{1}\right) & \Phi(-1) & \Phi\left(0_{1}\right) \\
a^{T} & 1 & 0 & 0 \\
-\bar{\Phi}(1)^{T} & 0 & 0 & -a_{1}^{-2} \\
-\bar{\Phi}(0)^{T} & 0 & -1 & a_{1}^{-1}
\end{array}\right| \\
& +\left|\begin{array}{cccc}
M_{d} & C\left(k_{1}+a_{1}\right) & \Phi\left(0_{1}\right) & \Phi\left(0_{2}\right) \\
a^{T} & 1 & 0 & 0 \\
-\bar{\Phi}(1)^{T} & 0 & -a_{1}^{-2} & -a_{2}^{-2} \\
-\bar{\Phi}(0)^{T} & 0 & a_{1}^{-1} & a_{2}^{-1}
\end{array}\right|\left|\begin{array}{ccc}
M_{d} & C\left(k_{1}\right) & \Phi(-1) \\
a^{T} & 1 & 0 \\
-\bar{\Phi}(0)^{T} & 0 & -1
\end{array}\right| \\
& -\left|\begin{array}{ccc}
M_{d} & \Phi(-1) & \Phi\left(0_{2}\right) \\
a^{T} & 1 & 0 \\
-\bar{\Phi}(0)^{T} & -1 & a_{2}^{-1}
\end{array}\right|\left|\begin{array}{cccc}
M_{d} & C\left(k_{1}+a_{1}\right) & \Phi\left(0_{1}\right) & C\left(k_{1}\right) \\
a^{T} & 1 & 0 & 1 \\
-\bar{\Phi}(1)^{T} & 0 & -a_{1}^{-2} & 0 \\
-\bar{\Phi}(0)^{T} & 0 & a_{1}^{-1} & 0
\end{array}\right|=0 .
\end{aligned}
$$


In order to show that determinant identity (2.28) holds, let us introduce the following $2(N+3) \times$ $2(N+3)$ determinant which vanishes,

$$
\left|\begin{array}{cccccccc}
M_{d} & 0 & 0 & 0 & 0 & C\left(k_{1}\right) & \Phi(-1) & \Phi\left(0_{2}\right) \\
\alpha^{T} & 0 & 0 & 0 & 0 & 1 & 0 & 0 \\
-\bar{\Phi}(1)^{T} & 0 & 0 & 0 & 1 & 0 & 0 & -a_{2}^{-2} \\
-\bar{\Phi}(0)^{T} & 0 & 0 & 0 & 0 & 0 & -1 & a_{2}^{-1} \\
0 & M_{d} & C\left(k_{1}+a_{1}\right) & \Phi\left(0_{1}\right) & 0 & C\left(k_{1}\right) & \Phi(-1) & \Phi\left(0_{2}\right) \\
0 & a^{T} & 1 & 0 & 0 & 1 & 0 & 0 \\
0 & -\bar{\Phi}(1)^{T} & 0 & -a_{1}^{-2} & 1 & 0 & 0 & -a_{2}^{-2} \\
0 & -\bar{\Phi}(0)^{T} & 0 & a_{1}^{-1} & 0 & 0 & -1 & a_{2}^{-1}
\end{array}\right|=0 .
$$

Applying the Laplace expansion in $(N+3) \times(N+3)$ minors to the left-hand side of (2.29), we obtain the determinant identity (2.28). Therefore, functions $f, g, P_{i, j}, Q_{i, j}$ given in equations (2.12.2,2.19-2.20) are solutions of equation (1.6). In the same way, substitution of equations (2.112.15,2.24-2.27) into equation (1.7) gives the determinant identity:

$$
\begin{aligned}
& -\left|\begin{array}{ccc}
M_{d} & C\left(k_{1}+a_{1}\right) \Phi\left(0_{1}\right) \\
\alpha^{T} & 1 & 0 \\
-\bar{\Phi}(0)^{T} & 0 & a_{1}^{-1}
\end{array}\right| \begin{array}{cccc}
M_{d} & C\left(k_{1}\right) & \Phi(-1) & \Phi\left(0_{3}\right) \\
\alpha^{T} & 1 & 0 & 0 \\
-\bar{\Phi}(1)^{T} & 0 & 0 & -a_{3}^{-2} \\
-\bar{\Phi}(0)^{T} & 0 & -1 & a_{3}^{-1}
\end{array} \mid \\
& +\left|\begin{array}{cccc}
M_{d} & C\left(k_{1}\right) & \Phi\left(0_{3}\right) \\
\alpha^{T} & 1 & 0 \\
-\bar{\Phi}(0)^{T} & 0 & a_{3}^{-1}
\end{array}\right|\left|\begin{array}{cccc}
M_{d} & C\left(k_{1}+a_{1}\right) & \Phi(-1) & \Phi\left(0_{1}\right) \\
\alpha^{T} & 1 & 0 & 0 \\
-\bar{\Phi}(1)^{T} & 0 & 0 & -a_{3}^{-2} \\
-\bar{\Phi}(0)^{T} & 0 & -1 & a_{3}^{-1}
\end{array}\right| \\
& +\left|\begin{array}{cccc}
M_{d} & C\left(k_{1}\right) & \Phi(-1) \\
\alpha^{T} & 1 & 0 \\
-\bar{\Phi}(0)^{T} & 0 & -1
\end{array}\right|\left|\begin{array}{cccc}
M_{d} & C\left(k_{1}+a_{1}\right) & \Phi\left(0_{1}\right) & \Phi\left(0_{3}\right) \\
\alpha^{T} & 1 & 0 & 0 \\
-\bar{\Phi}(1)^{T} & 0 & -a_{1}^{-2} & -a_{3}^{-2} \\
-\bar{\Phi}(0)^{T} & 0 & a_{1}^{-1} & a_{3}^{-1}
\end{array}\right| \\
& -\left|\begin{array}{cccc}
M_{d} & C\left(k_{1}+a_{1}\right) & \Phi\left(0_{1}\right) & C\left(k_{1}\right) \\
\alpha^{T} & 1 & 0 & 1 \\
M_{d} & \Phi(-1) & \Phi\left(0_{3}\right) \\
\alpha^{T} & 1 & 0 \\
-\bar{\Phi}(0)^{T} & -1 & a_{3}^{-1}
\end{array}\right| \begin{array}{cccc}
-\bar{\Phi}(0)^{T} & 0 & -a_{1}^{-2} & 0 \\
-\bar{\Phi}(0)^{T} & 0 & a_{1}^{-1} & 0
\end{array} \mid=0
\end{aligned}
$$

which can also be proved similarly.

Now we prove $f, g$ given in (2.1-2.2) and $P_{i, j}, Q_{i, j}$ for $r, m=1, \cdots, K$ given in (2.19-2.20) are solutions to equations (2.22-2.23). Substituting equations (2.11-2.15,2.19,2.24-2.27) into equations (2.22-2.23), we get the following two identities for the determinant, respectively:

$$
\begin{array}{r}
-\left|\begin{array}{ccc}
M_{d} & C\left(k_{1}\right) \Phi\left(0_{2}\right) \\
\alpha^{T} & 1 & 0 \\
-\Psi(0)^{T} & 0 & a_{2}^{-1}
\end{array}\right|\left|\begin{array}{ccc}
D_{i j} & R_{i j} & \Phi_{i}\left(0_{3}\right) \\
-\bar{\Phi}_{j}(1)^{T} & -\bar{\phi}_{j}(1) & -a_{3}^{-2} \\
-\bar{\Phi}_{j}(0)^{T} & -\bar{\phi}_{j}(0) & a_{3}^{-1}
\end{array}\right| \\
+\left|\begin{array}{ccc}
M_{d} & C\left(k_{1}\right) & \Phi\left(0_{3}\right) \\
\alpha^{T} & 1 & 0 \\
-\bar{\Phi}(0)^{T} & 0 & a_{3}^{-1}
\end{array}\right|\left|\begin{array}{ccc}
D_{i j} & R_{i j} & \Phi_{i}\left(0_{2}\right) \\
-\bar{\Phi}_{j}(1)^{T} & -\bar{\phi}_{j}(1) & -a_{2}^{-2} \\
-\bar{\Phi}_{j}(0)^{T} & -\bar{\phi}_{j}(0) & a_{2}^{-1}
\end{array}\right|
\end{array}
$$


and

$$
+\left|\begin{array}{cccc}
M_{d} & C\left(k_{1}\right) & \Phi\left(0_{2}\right) \Phi\left(0_{3}\right) \\
\alpha^{T} & 1 & 0 & 0 \\
-\bar{\Phi}(1)^{T} & 0 & -a_{2}^{-2} & -a_{3}^{-2} \\
-\bar{\Phi}(0)^{T} & 0 & a_{2}^{-1} & a_{3}^{-1}
\end{array}\right|\left|\begin{array}{cc}
D_{i j} & R_{i j} \\
-\bar{\Phi}_{j}(0)^{T} & -\bar{\phi}_{j}(0)
\end{array}\right|=0
$$

$$
\begin{aligned}
& \left|\begin{array}{ccc}
D_{i j} & \Phi_{i}(-1) & \Phi_{i}\left(0_{2}\right) \\
-\bar{\Phi}_{j}(0)^{T} & -1 & a_{2}^{-1} \\
M_{i j}^{T} & \phi_{i}(-1) & \phi_{i}\left(0_{2}\right)
\end{array}\right|\left|\begin{array}{cccc}
M_{d} & C\left(k_{1}\right) \Phi(-1) & \Phi\left(0_{3}\right) \\
\alpha^{T} & 1 & 0 & 0 \\
-\bar{\Phi}(1)^{T} & 0 & 0 & -a_{3}^{-2} \\
-\bar{\Phi}(0)^{T} & 0 & -1 & a_{3}^{-1}
\end{array}\right| \\
& -\left|\begin{array}{ccc}
D_{i j} & \Phi_{i}(-1) & \Phi_{i}\left(0_{3}\right) \\
-\bar{\Phi}_{j}(0)^{T} & -1 & a_{3}^{-1} \\
M_{i j}^{T} & \phi_{i}(-1) & \phi_{i}\left(0_{3}\right)
\end{array}\right|\left|\begin{array}{cccc}
M_{d} & C\left(k_{1}\right) & \Phi(-1) & \Phi\left(0_{2}\right) \\
\alpha^{T} & 1 & 0 & 0 \\
-\bar{\Phi}(1)^{T} & 0 & 0 & -a_{2}^{-2} \\
-\bar{\Phi}(0)^{T} & 0 & -1 & a_{2}^{-1}
\end{array}\right| \\
& -\left|\begin{array}{cccc}
D_{i j} & \Phi_{i}(-1) & \Phi_{i}\left(0_{2}\right) & \Phi_{i}\left(0_{3}\right) \\
-\bar{\Phi}_{j}(1)^{T} & 0 & -a_{2}^{-2} & -a_{3}^{-2} \\
M_{i j}^{T} & \phi_{i}(-1) & \phi_{i}\left(0_{2}\right) & \phi_{i}\left(0_{3}\right) \\
-\bar{\Phi}_{j}(0)^{T} & -1 & a_{2}^{-1} & a_{3}^{-1}
\end{array}\right|\left|\begin{array}{ccc}
M_{d} & C\left(k_{1}\right) & \Phi(-1) \\
\alpha^{T} & 1 & 0 \\
-\bar{\Phi}(0)^{T} & 0 & -1
\end{array}\right|=0 .
\end{aligned}
$$

Now we prove the determinant identity (2.31). Let us introduce the following $2(N+2) \times 2(N+$ 2) determinant which is equal to zero:

$$
\left|\begin{array}{cccccc}
D_{i}^{R} & \Phi_{i}\left(0_{2}\right) & 0 & \Phi_{i}\left(0_{3}\right) & 0 & 0 \\
M_{i} & \phi_{i}\left(0_{2}\right) & 0 & \phi_{i}\left(0_{3}\right) & 0 & 0 \\
-\bar{\Phi}(1)^{T} & -a_{2}^{-2} & 1 & -a_{3}^{-2} & 0 & 0 \\
-\bar{\Phi}(0)^{T} & a_{2}^{-1} & 0 & a_{3}^{-1} & 0 & 0 \\
0 & \Phi_{i}\left(0_{2}\right) & 0 & \Phi_{i}\left(0_{3}\right) & D_{i}^{R} & 0 \\
0 & \phi_{i}\left(0_{2}\right) & 0 & \phi_{i}\left(0_{3}\right) & M_{i} & 1 \\
0 & -a_{2}^{-2} & 1 & -a_{3}^{-2} & -\bar{\Phi}(1)^{T} & 0 \\
0 & a_{2}^{-1} & 0 & a_{3}^{-1} & -\bar{\Phi}(0)^{T} & 0
\end{array}\right|=0
$$

where $D_{i}^{R}$ denotes the $(N-1) \times N$ matrix obtained by eliminating the $i$ th row from the matrix $\left(c_{i, j}\left(k_{1}\right)+m_{i j}\left(k_{1}, k_{2}, k_{3}\right)\right)_{1 \leq i, j \leq N}$, and $M_{i}$ is given by

$$
M_{i}=\left(c_{i}\left(k_{1}\right)+m_{i 1}, \cdots, c_{i}\left(k_{1}\right)+m_{i, K}, d_{i, K+1}+m_{i, K+1}, \cdots, d_{i, N}+m_{i, N}\right)^{T} .
$$

Applying the Laplace expansion in $(N+2) \times(N+2)$ minors to the left-hand side of equation (2.33), we obtain the determinant identity (2.31).

Similarly, the Laplace expansion of the following $2(N+2) \times 2(N+2)$ determinant which is equal to zero in $(N+2) \times(N+2)$ minors:

$$
\left|\begin{array}{cccccccc}
D_{j}^{C} & \Phi(-1) & 0 & 0 & 0 & 0 & \Phi\left(0_{2}\right) & \Phi\left(0_{3}\right) \\
-\bar{\Phi}_{j}(1)^{T} & 0 & 1 & 0 & 0 & 0 & -a_{2}^{-2} & -a_{3}^{-2} \\
-\bar{\Phi}(0)^{T} & -1 & 0 & 0 & 0 & 0 & a_{2}^{-1} & a_{3}^{-1} \\
0 & 0 & 0 & \Phi(-1) & D_{j}^{C} & R_{j} & \Phi\left(0_{2}\right. & \Phi\left(0_{3}\right) \\
0 & 0 & 1 & 0 & -\bar{\Phi}_{j}(1)^{T} & -\bar{\phi}_{j}(1) & -a_{2}^{-2} & -a_{3}^{-2} \\
0 & 0 & 0 & -1 & -\bar{\Phi}_{j}(0)^{T} & -\bar{\phi}_{j}(0) & a_{2}^{-1} & a_{3}^{-1}
\end{array}\right|=0
$$


where $D_{j}^{C}$ denotes the $N \times(N-1)$ matrix obtained by eliminating the $j$ th column from the matrix $\left(c_{i, j}\left(k_{1}\right)+m_{i j}\left(k_{1}, k_{2}, k_{3}\right)\right)_{1 \leq i, j \leq N}$, and $R_{j}$ is given by

$$
R_{j}=\left(c_{1}\left(k_{1}\right)+m_{1 j}, \cdots, c_{K}\left(k_{1}\right)+m_{K, j K}, d_{K+1, j}+m_{K+1, j}, \cdots, d_{N, j}+m_{N, j}\right)^{T},
$$

yields the determinant identity (2.32).

So functions (2.1-2.2) and (2.19-2.20) for $r, m=1, \cdots, K$ are solutions to the modified discrete $\mathrm{KP}$ equation with self-consistent sources $(2.17-2.18,2.22-2.23)$.

If we choose the arbitrary function $c_{i j}$ in (2.10) as a function of $k_{2}$ instead of $k_{1}$, we can produce another form of the modified discrete KP equation with self-consistent sources via source generation procedure in a similar way:

$$
\begin{aligned}
& a_{1} f\left(k_{1}+a_{1}\right) g\left(k_{2}+a_{2}\right)-a_{2} f\left(k_{2}+a_{2}\right) g\left(k_{1}+a_{1}\right)+\left(a_{2}-a_{1}\right) f\left(k_{1}+a_{1}, k_{2}+a_{2}\right) g\left(k_{1}, k_{2}, k_{3}\right) \\
& =a_{1} \sum_{i, j=1}^{K} P_{i, j}\left(k_{2}+a_{2}\right) Q_{i, j}\left(k_{1}+a_{1}\right), \\
& a_{1} f\left(k_{1}+a_{1}\right) g\left(k_{3}+a_{3}\right)-a_{3} f\left(k_{3}+a_{3}\right) g\left(k_{1}+a_{1}\right)+\left(a_{3}-a_{1}\right) f\left(k_{1}+a_{1}, k_{3}+a_{3}\right) g\left(k_{1}, k_{2}, k_{3},\right) \\
& =0, \\
& a_{1} f\left(k_{1}+a_{1}\right) P_{i j}\left(k_{3}+a_{3}\right)-a_{3} f\left(k_{3}+a_{3}\right) P_{i j}\left(k_{1}+a_{1}\right)+\left(a_{3}-a_{1}\right) f\left(k_{1}+a_{1}, k_{3}+a_{3}\right) P_{i j}\left(k_{1}, k_{2}, k_{3}\right) \\
& =0, \\
& a_{1} Q_{i j}\left(k_{1}+a_{1}\right) g\left(k_{3}+a_{3}\right)-a_{3} Q_{i j}\left(k_{3}+a_{3}\right) g\left(k_{1}+a_{1}\right)+\left(a_{3}-a_{1}\right) Q_{i j}\left(k_{1}+a_{1}, k_{3}+a_{3}\right) g\left(k_{1}, k_{2}, k_{3}\right) \\
& =0 .
\end{aligned}
$$

Equations (2.34-2.37) can be written in a compact way using the bilinear operator (1.3):

$$
\begin{gathered}
{\left[a_{1} e^{\frac{1}{2} a_{1} D_{k_{1}}-\frac{1}{2} a_{2} D_{k_{2}}}-a_{2} e^{\frac{1}{2} a_{2} D_{k_{2}}-\frac{1}{2} a_{1} D_{k_{1}}}+\left(a_{2}-a_{1}\right) e^{\frac{1}{2} a_{1} D_{k_{1}}+\frac{1}{2} a_{2} D_{k_{2}}}\right] f \cdot g} \\
=a_{1} \sum_{i, j=1}^{K} e^{\frac{1}{2} a_{2} D_{k_{2}}-\frac{1}{2} a_{1} D_{k_{1}} P_{i j} \cdot Q_{i j},} \\
{\left[a_{1} e^{\frac{1}{2} a_{1} D_{k_{1}}-\frac{1}{2} a_{3} D_{k_{3}}}-a_{3} e^{\frac{1}{2} a_{3} D_{k_{3}}-\frac{1}{2} a_{1} D_{k_{1}}}+\left(a_{3}-a_{1}\right) e^{\frac{1}{2} a_{1} D_{k_{1}}+\frac{1}{2} a_{3} D_{k_{3}}}\right] f \cdot g=0,} \\
{\left[a_{1} e^{\frac{1}{2} a_{1} D_{k_{1}}-\frac{1}{2} a_{3} D_{k_{3}}}-a_{3} e^{\frac{1}{2} a_{3} D_{k_{3}}-\frac{1}{2} a_{1} D_{k_{1}}}+\left(a_{3}-a_{1}\right) e^{\frac{1}{2} a_{1} D_{k_{1}}+\frac{1}{2} a_{3} D_{k_{3}}}\right] f \cdot P_{i j}=0,} \\
{\left[a_{1} e^{\frac{1}{2} a_{1} D_{k_{1}}-\frac{1}{2} a_{3} D_{k_{3}}}-a_{3} e^{\frac{1}{2} a_{3} D_{k_{3}}-\frac{1}{2} a_{1} D_{k_{1}}}+\left(a_{3}-a_{1}\right) e^{\frac{1}{2} a_{1} D_{k_{1}}+\frac{1}{2} a_{3} D_{k_{3}}}\right] Q_{i j} \cdot g=0 .}
\end{gathered}
$$

We can derive the following discrete Gram-type determinant solution for the modified discrete KP equation with self-consistent sources (2.34-2.37) or (2.38-2.41):

$$
\begin{gathered}
f\left(k_{1}, k_{2}, k_{3}\right)=\operatorname{det}\left(\bar{c}_{i j}\left(k_{2}\right)+m_{i j}\right)_{1 \leq i, j \leq N}=|\bar{D}|, \\
g\left(k_{1}, k_{2}, k_{3}\right)=\left|\begin{array}{cc}
\bar{D} & \Phi(-1) \\
\bar{\Phi}(0)^{T} & 1
\end{array}\right|
\end{gathered}
$$

where $m_{i j}(1 \leq i, j \leq N), \Phi(s), \bar{\Phi}(s)$ are given in (2.3-2.7). In addition, $\bar{c}_{i j}\left(k_{2}\right)$ satisfies

$$
\bar{c}_{i j}\left(k_{2}\right)=\left\{\begin{array}{lc}
\bar{c}_{i}\left(k_{2}\right), & 1 \leq i \leq K \leq N \text { and } j=1,2, \cdots, K, K \in Z^{+}, \\
\bar{d}_{i j}, & \text { otherwise }
\end{array}\right.
$$


with $\bar{c}_{i}\left(k_{2}\right)(1 \leq i \leq K)$ being an arbitrary function of $k_{2}, \bar{d}_{i j}(1 \leq i, j \leq N)$ being an arbitrary constant and $K$ being a positive integer, and

$$
\begin{gathered}
P_{i, j}\left(k_{1}, k_{2}, k_{3}\right)=\sqrt{c_{i}\left(k_{2}\right)-c_{i}\left(k_{2}-a_{2}\right)}\left|\begin{array}{cc}
\bar{D}_{i j} & \bar{R}_{i j} \\
\bar{\Phi}_{j}(0)^{T} & \bar{\phi}_{j}(0)
\end{array}\right|, \\
Q_{i, j}\left(k_{1}, k_{2}, k_{3}\right)=a_{1} \sqrt{\Delta c_{i}\left(k_{2}\right)}\left|\begin{array}{cc}
\bar{D}_{i j} & \Phi_{i}(-1) \\
\bar{M}_{i j}^{T} & \phi_{i}(-1)
\end{array}\right|,
\end{gathered}
$$

where $\bar{D}_{i j}$ is the $(N-1)$ th order matrice obtained by eliminating the $i$ th row and the $j$ th column from the $N \times N$ matrice $\bar{D}$, and

$$
\begin{gathered}
\bar{R}_{i j}=\left(\bar{c}_{1}\left(k_{2}\right)+m_{1 j}, \cdots, \bar{c}_{i-1}\left(k_{2}\right)+m_{i-1, j}, \bar{c}_{i+1}\left(k_{2}\right)+m_{i+1, j}, \cdots,\right. \\
\left.\bar{c}_{K}\left(k_{2}\right)+m_{K, j}, \bar{d}_{K+1, j}+m_{K+1, j}, \cdots, \bar{d}_{N, j}+m_{N, j}\right)^{T}, \\
\bar{M}_{i j}=\left(\bar{c}_{i}\left(k_{2}\right)+m_{i 1}, \cdots, \bar{c}_{i}\left(k_{2}\right)+m_{i, j-1}, \bar{c}_{i}\left(k_{2}\right)+m_{i, j+1}, \cdots,\right. \\
\left.\bar{c}_{i}\left(k_{2}\right)+m_{i, K}, \bar{d}_{i, K+1}+m_{i, K+1}, \cdots, \bar{d}_{i, N}+m_{i, N}\right)^{T} .
\end{gathered}
$$

\section{Commutativity of the source generation procedure and Bäcklund transformation}

In this section, we show that the commutativity of the source generation procedure and Bäcklund transformation holds for the discrete KP equation. For this purpose, we derive another form of the modified discrete KP with self-consistent sources which is the Bäcklund transformation for the discrete KPequation with self-consistent sources.

We has shown that the Grammian determinants $f\left(k_{1}, k_{2}, k_{3}\right), g\left(k_{1}, k_{2}, k_{3}\right), P_{i, j}\left(k_{1}, k_{2}, k_{3}\right)$, $Q_{i, j}\left(k_{1}, k_{2}, k_{3}\right)$ given in (2.42-2.45) satisfy the modified discrete KP equation with self-consistent sources (2.38-2.41). Now we take

$$
\begin{aligned}
& f^{\prime}\left(k_{1}, k_{2}, k_{3}\right)=g\left(k_{1}, k_{2}, k_{3}\right), \\
& g_{i, j}^{\prime}\left(k_{1}, k_{2}, k_{3}\right)=Q_{i j}\left(k_{1}, k_{2}, k_{3}\right), \\
& h_{i, j}\left(k_{1}, k_{2}, k_{3}\right)=P_{i, j}\left(k_{1}, k_{2}, k_{3}\right),
\end{aligned}
$$

and introduce two new fields

$$
\begin{gathered}
g_{i, j}\left(k_{1}, k_{2}, k_{3}\right)=-a_{1} a_{3} \sqrt{\Delta c_{i}\left(k_{2}\right)}\left|\bar{D}_{j}^{C} \Phi\left(k_{3}\right)\right|, \\
h_{i, j}^{\prime}\left(k_{1}, k_{2}, k_{3}\right)=-a_{3} \sqrt{c_{i}\left(k_{2}\right)-c_{i}\left(k_{2}-a_{2}\right)}\left|\begin{array}{cc}
\bar{D}_{i}^{R} & \Phi_{i}(-1) \\
\bar{\Phi}(1)^{T} & 0 \\
\bar{\Phi}(0)^{T} & 1
\end{array}\right|,
\end{gathered}
$$

where $\bar{D}_{j}^{C}$ denotes the $N \times(N-1)$ matrix obtained by eliminating the $j$ th column from the $N \times N$ matrice $\bar{D}$ and $\bar{D}_{i}^{R}$ denotes the $(N-1) \times N$ matrix obtained by eliminating the $i$ th row from the $N \times N$ matrice $\bar{D}$. 
It is not difficult to show that Grammian determinants $f, g_{i, j}, h_{i, j}$ and $f^{\prime}, g_{i, j}^{\prime}, h_{i, j}^{\prime}$ are two solutions to the discrete KP equation with self-consistent sources derived in [23]:

$$
\begin{aligned}
& \left(a_{1}\left(a_{2}-a_{3}\right) e^{1 / 2\left(-D_{k_{1}}+D_{k_{2}}+D_{k_{3}}\right)}+a_{2}\left(a_{3}-a_{1}\right) e^{1 / 2\left(D_{k_{1}}-D_{k_{2}}+D_{k_{3}}\right)}+a_{3}\left(a_{1}-a_{2}\right) e^{1 / 2\left(-D_{k_{1}}-D_{k_{2}}+D_{k_{3}}\right)}\right) f \cdot f \\
& =\sum_{i, j=1}^{K} e^{1 / 2\left(D_{k_{1}}-D_{k_{2}}+D_{k_{3}}\right)} g_{i, j} \cdot h_{i, j} \\
& \left(a_{3} e^{1 / 2\left(D_{k_{3}}-D_{k_{1}}\right)}-a_{1} e^{1 / 2\left(D_{k_{1}}-D_{k_{3}}\right)}+\left(a_{1}-a_{3}\right) e^{1 / 2\left(D_{k_{1}}+D_{k_{3}}\right)}\right) f \cdot h_{i, j}=0, \quad j=1, \cdots, K \\
& \left(a_{3} e^{1 / 2\left(D_{k_{3}}-D_{k_{1}}\right)}-a_{1} e^{1 / 2\left(D_{k_{1}}-D_{k_{3}}\right)}+\left(a_{1}-a_{3}\right) e^{1 / 2\left(D_{k_{1}}+D_{k_{3}}\right)}\right) g_{i, j} \cdot f=0, \quad j=1, \cdots, K
\end{aligned}
$$

Furthermore, we can verify that the Grammian determinants $f, f^{\prime}, g_{i, j}, g_{i, j}^{\prime}, h_{i, j}, h_{i, j}^{\prime}$ satisfy the following six bilinear equations:

$$
\begin{aligned}
& {\left[a_{1} e^{\frac{1}{2} D_{k_{1}}-\frac{1}{2} D_{k_{2}}}-a_{2} e^{\frac{1}{2} D_{k_{2}}-\frac{1}{2} D_{k_{1}}}+\left(a_{2}-a_{1}\right) e^{\frac{1}{2} D_{k_{1}}+\frac{1}{2} D_{k_{2}}}\right] f \cdot f^{\prime}=\sum_{i, j=1}^{K} e^{\frac{1}{2} D_{k_{2}}-\frac{1}{2} D_{k_{1}}} h_{i j} \cdot g_{i j}^{\prime},} \\
& {\left[a_{1} e^{\frac{1}{2} D_{k_{1}}-\frac{1}{2} D_{k_{3}}}-a_{3} e^{\frac{1}{2} D_{k_{3}}-\frac{1}{2} D_{k_{1}}}+\left(a_{3}-a_{1}\right) e^{\frac{1}{2} D_{k_{1}}+\frac{1}{2} D_{k_{3}}}\right] f \cdot f^{\prime}=0,} \\
& {\left[a_{1} e^{\frac{1}{2} D_{k_{1}}-\frac{1}{2} D_{k_{3} 2}}-a_{3} e^{\frac{1}{2} D_{k_{3}}-\frac{1}{2} D_{k_{1}}}+\left(a_{3}-a_{1}\right) e^{\frac{1}{2} D_{k_{1}}+\frac{1}{2} D_{k_{3}}}\right] g_{i, j} \cdot g_{i, j}^{\prime}=0, \quad j=1, \cdots, K,} \\
& {\left[a_{1} e^{\frac{1}{2} D_{k_{1}}-\frac{1}{2} D_{k_{3}}}-a_{3} e^{\frac{1}{2} D_{k_{3}}-\frac{1}{2} D_{k_{1}}}+\left(a_{3}-a_{1}\right) e^{\frac{1}{2} D_{k_{1}}+\frac{1}{2} D_{k_{3}}}\right] h_{i, j} \cdot h_{i, j}^{\prime}=0, \quad j=1, \cdots, K,,} \\
& e^{\frac{1}{2} D_{k_{3}}} g_{i, j} \cdot f^{\prime}=\left(e^{-\frac{1}{2} D_{k_{3}}}-e^{\frac{1}{2} D_{k_{3}}}\right) f \cdot g_{i, j}^{\prime}, \quad j=1, \cdots, K, \\
& e^{\frac{1}{2} D_{k_{3}}} f \cdot h_{i, j}^{\prime}=\left(e^{-\frac{1}{2} D_{k_{3}}}-e^{\frac{1}{2} D_{k_{3}}}\right) h_{i, j} \cdot f^{\prime}, \quad j=1, \cdots, K .
\end{aligned}
$$

Equations (3.9-3.14) constitute another form of the modified discrete KP with self-consistent sources. It is proved in [23] that the discrete KP equation with self-consistent sources (3.6-3.8) possesses the following bilinear Bäcklund transformation:

$$
\begin{aligned}
& {\left[\gamma e^{\frac{1}{2} D_{k_{1}}-\frac{1}{2} D_{k_{2}}}+z_{2} e^{\frac{1}{2} D_{k_{2}}-\frac{1}{2} D_{k_{1}}}+\lambda_{1} z_{3} e^{\frac{1}{2} D_{k_{1}}+\frac{1}{2} D_{k_{2}}}\right] f \cdot f^{\prime}=\lambda_{2} \sum_{i, j=1}^{K} e^{\frac{1}{2} D_{k_{2}}-\frac{1}{2} D_{k_{1}}} h_{i j} \cdot g_{i j}^{\prime},} \\
& {\left[\beta_{1} e^{\frac{1}{2} D_{k_{1}}-\frac{1}{2} D_{k_{3}}}-e^{\frac{1}{2} D_{k_{3}}-\frac{1}{2} D_{k_{1}}}+\lambda_{1} e^{\frac{1}{2} D_{k_{1}}+\frac{1}{2} D_{k_{3}}}\right] f \cdot f^{\prime}=0,} \\
& {\left[\beta_{1} e^{\frac{1}{2} D_{k_{1}}-\frac{1}{2} D_{k_{3}}}-e^{\frac{1}{2} D_{k_{3}}-\frac{1}{2} D_{k_{1}}}+\lambda_{1} e^{\frac{1}{2} D_{k_{1}}+\frac{1}{2} D_{k_{3}}}\right] g_{i, j} \cdot g_{i, j}^{\prime}=0, \quad j=1, \cdots, K,} \\
& {\left[\beta_{1} e^{\frac{1}{2} D_{k_{1}}-\frac{1}{2} D_{k_{3}}}-e^{\frac{1}{2} D_{k_{3}}-\frac{1}{2} D_{k_{1}}}+\lambda_{1} e^{\frac{1}{2} D_{k_{1}}+\frac{1}{2} D_{k_{3}}}\right] h_{i, j} \cdot h_{i, j}^{\prime}=0, \quad j=1, \cdots, K,} \\
& e^{\frac{1}{2} D_{k_{3}}} g_{i, j} \cdot f^{\prime}=\left(\beta_{2} e^{-\frac{1}{2} D_{k_{3}}}+\lambda_{2} e^{\frac{1}{2} D_{k_{3}}}\right) f \cdot g_{i, j}^{\prime}, \quad j=1, \cdots, K,
\end{aligned}
$$

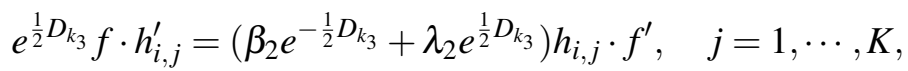

where $\gamma$ is an arbitrary constant and $\beta_{1}, \beta_{2}, \lambda_{1}, \lambda_{2}$ are constants satisfying the relation $\lambda_{1} \beta_{2} a_{1}=$ $\lambda_{2} \beta_{1}\left(a_{1}-a_{3}\right)$.

If we take $\gamma=-a_{1}, \lambda_{1}=\frac{a_{3}-a_{1}}{a_{3}}, \lambda_{2}=-1, \beta_{1}=\frac{a_{1}}{a_{3}}, \beta_{2}=1$, then equations (3.15-3.20) become (3.9-3.14). Hence the modified discrete KP with self-consistent sources (3.9-3.14) is a Bäcklund transformation for the discrete KP equation with self-consistent sources (3.6-3.8).

\section{Summary and discussion}

In this paper, we have produced two forms of the the modified discrete KP equation with selfconsistent sources (2.17-2.23) and (2.38-2.41) via the source generation procedure, and given their 
discrete Gram-type determinant solutions. We have also constructed another form of the modified discrete KP equation with self-consistent sources (3.9-3.14) which is the Bäcklund transformation for the discrete KP equation with self-consistent sources derived in [23].

We investigate a continuum limit of the modified discrete KP with self-consistent sources (2.342.37) with one pair of sources $P_{11}\left(k_{1}, k_{2}, k_{3}\right), Q_{11}\left(k_{1}, k_{2}, k_{3}\right)$, for simplicity. If we take

$$
\begin{aligned}
& a_{1}=\delta, a_{2}=\varepsilon, a_{3}=1, D_{k_{1}}=D_{y}, D_{k_{2}}=D_{y}-\frac{1}{2} \varepsilon D_{t}, D_{k_{3}}=D_{n}, \\
& f\left(k_{1}, k_{2}, k_{3}\right)=F(n, y, t), g\left(k_{1}, k_{2}, k_{3}\right)=G(n, y, t), \\
& P_{11}\left(k_{1}, k_{2}, k_{3}\right)=\varepsilon S_{11}(n, y, t), Q_{11}\left(k_{1}, k_{2}, k_{3}\right)=\frac{1}{2} \varepsilon H_{11}(n, y, t),
\end{aligned}
$$

in (2.38-2.41) with one pair of sources $P, Q$ and compare $\varepsilon^{2} \delta$ order of the two sides of the equation (2.38) and $\delta$ order of the two sides of the equations (2.39-2.41), then we obtain the modified differential-difference KP equation with self-consistent sources:

$$
\begin{aligned}
& \left(D_{y}^{2}+D_{t}\right) F \cdot G=H_{11} S_{11}, \\
& D_{y} e^{\frac{1}{2} D_{n}} F \cdot G=\left(e^{\frac{1}{2} D_{n}}-e^{-\frac{1}{2} D_{n}}\right) F \cdot G, \\
& D_{y} e^{\frac{1}{2} D_{n}} F \cdot S_{11}=\left(e^{\frac{1}{2} D_{n}}-e^{-\frac{1}{2} D_{n}}\right) F \cdot S_{11}, \\
& D_{y} e^{\frac{1}{2} D_{n}} H_{11} \cdot G=\left(e^{\frac{1}{2} D_{n}}-e^{-\frac{1}{2} D_{n}}\right) H_{11} \cdot G,
\end{aligned}
$$

which can be derived by applying source generation procedure to the modified differentialdifference KP equation [25]:

$$
\begin{aligned}
& \left(D_{y}^{2}+D_{t}\right) F \cdot G=0, \\
& D_{y} e^{\frac{1}{2} D_{n}} F \cdot G=\left(e^{\frac{1}{2} D_{n}}-e^{-\frac{1}{2} D_{n}}\right) F \cdot G .
\end{aligned}
$$

Now we show that the exact solution to the modified discrete KP with self-consistent sources (2.34-2.37) yields the exact solution to the modified differential-difference KP equation with selfconsistent sources (4.4-4.7) through the continuum limit.

We obtain the N-soliton solution for the modified discrete KP with self-consistent sources (2.342.37) by choosing

$$
\begin{aligned}
& m_{i, j}\left(k_{1}, k_{2}, k_{3}\right)=\frac{1}{p_{i}+\bar{p}_{j}}\left(\frac{1+\bar{p}_{j} a_{1}}{1-p_{i} a_{1}}\right)^{\frac{k_{1}}{a_{1}}}\left(\frac{1+\bar{p}_{j} a_{2}}{1-p_{i} a_{2}}\right)^{\frac{k_{2}}{a_{2}}}\left(\frac{1+\bar{p}_{j} a_{3}}{1-p_{i} a_{3}}\right)^{\frac{k_{3}}{a_{3}}}, 1 \leq i, j \leq N, \\
& \phi_{i}\left(k_{1}, k_{2}, k_{3} ; s\right)=p_{i}^{s}\left(1-p_{i} a_{1}\right)^{-\frac{k_{1}}{a_{1}}}\left(1-p_{i} a_{2}\right)^{-\frac{k_{2}}{a_{2}}}\left(1-p_{i} a_{3}\right)^{-\frac{k_{3}}{a_{3}}}, 1 \leq i \leq N, \\
& \bar{\phi}_{j}\left(k_{1}, k_{2}, k_{3} ; s\right)=\bar{p}_{j}^{s}\left(1+\bar{p}_{j} a_{1}\right)^{-\frac{k_{1}}{a_{1}}}\left(1+\bar{p}_{j} a_{2}\right)^{-\frac{k_{2}}{a_{2}}}\left(1+\bar{p}_{j} a_{3}\right)^{-\frac{k_{3}}{a_{3}}}, 1 \leq j \leq N,
\end{aligned}
$$

in equations (2.42-2.45). In equations (4.8-4.10), $p_{i}, \bar{p}_{i}(1 \leq i \leq N)$ are arbitrary constants. For example, if we take $N=1, K=1$ and

$$
\begin{aligned}
& \phi_{1}\left(k_{1}, k_{2}, k_{3} ; s\right)=p_{1}^{s}\left(1-p_{1} a_{1}\right)^{-\frac{k_{1}}{a_{1}}}\left(1-p_{1} a_{2}\right)^{-\frac{k_{2}}{a_{2}}}\left(1-p_{1} a_{3}\right)^{-\frac{k_{3}}{a_{3}}}, \\
& \bar{\phi}_{j}\left(k_{1}, k_{2}, k_{3} ; s\right)=\bar{p}_{1}^{s}\left(1-\bar{p}_{1} a_{1}\right)^{-\frac{k_{1}}{a_{1}}}\left(1-\bar{p}_{1} a_{2}\right)^{-\frac{k_{2}}{a_{2}}}\left(1-\bar{p}_{1} a_{3}\right)^{-\frac{k_{3}}{a_{3}}}, \\
& c_{1}\left(k_{2}\right)=\frac{\left(1+2 a_{2} \beta_{1}\left(k_{2}\right)\right)^{\frac{1}{a_{2}}}}{p_{1}+\bar{p}_{1}},
\end{aligned}
$$


where $\beta_{1}\left(k_{2}\right)$ is an arbitrary function of $k_{2}$, we obtain the one-soliton solution of the modified discrete KP with self-consistent sources (2.34-2.37):

$$
\begin{aligned}
& f=\frac{\left(1+2 a_{2} \beta_{1}\left(k_{2}\right)\right)^{\frac{1}{a_{2}}}}{p_{1}+\bar{p}_{1}}+\frac{1}{p_{1}+\bar{p}_{1}}\left(\frac{1+\bar{p}_{1} a_{1}}{1-p_{1} a_{1}}\right)^{\frac{k_{1}}{a_{1}}}\left(\frac{1+\bar{p}_{1} a_{2}}{1-p_{1} a_{2}}\right)^{\frac{k_{2}}{a_{2}}}\left(\frac{1+\bar{p}_{1} a_{3}}{1-p_{1} a_{3}}\right)^{\frac{k_{3}}{a_{3}}}, \\
& g=\frac{\left(1+2 a_{2} \beta_{1}\left(k_{2}\right)\right)^{\frac{1}{a_{2}}}}{p_{1}+\bar{p}_{1}}-\frac{\bar{p}_{1}}{p_{1}\left(p_{1}+\bar{p}_{1}\right)}\left(\frac{1+\bar{p}_{1} a_{1}}{1-p_{1} a_{1}}\right)^{\frac{k_{1}}{a_{1}}}\left(\frac{1+\bar{p}_{1} a_{2}}{1-p_{1} a_{2}}\right)^{\frac{k_{2}}{a_{2}}}\left(\frac{1+\bar{p}_{1} a_{3}}{1-p_{1} a_{3}}\right)^{\frac{k_{3}}{a_{3}}}, \\
& P_{11}=\sqrt{c_{1}\left(k_{2}\right)-c_{1}\left(k_{2}-a_{2}\right)\left(1+\bar{p}_{1} a_{1}\right)^{\frac{k_{1}}{a_{1}}}\left(1+\bar{p}_{1} a_{2}\right)^{\frac{k_{2}}{a_{2}}}\left(1+\bar{p}_{1} a_{3}\right)^{\frac{k_{3}}{a_{3}}},} \\
& Q_{11}=\frac{1}{p_{1}} \sqrt{c_{1}\left(k_{2}+a_{2}\right)-c_{1}\left(k_{2}\right)}\left(1-p_{1} a_{1}\right)^{-\frac{k_{1}}{a_{1}}}\left(1-p_{1} a_{2}\right)^{-\frac{k_{2}}{a_{2}}}\left(1-p_{1} a_{3}\right)^{-\frac{k_{3}}{a_{3}}} .
\end{aligned}
$$

We derive the following variable transformation from equation (4.1):

$$
t=-\frac{1}{2} \varepsilon k_{2}, \quad y=k_{1}+k_{2}, \quad n=k_{3} .
$$

Applying equations (4.18) and setting $\beta_{1}\left(k_{2}\right)=b_{1}(t), c_{1}\left(k_{2}\right)=C_{1}(t)$, we obtain

$$
c_{1}\left(k_{2}\right)=\frac{\left(1+2 a_{2} \beta_{1}\left(k_{2}\right)\right)^{\frac{1}{a_{2}}}}{p_{1}+\bar{p}_{1}}=\frac{e^{\frac{1}{\varepsilon} \ln \left(1+2 \varepsilon \beta_{1}\left(k_{2}\right)\right.}}{p_{1}+\bar{p}_{1}}=\frac{e^{2 \beta_{1}\left(k_{2}\right)+O(\varepsilon)}}{p_{1}+\bar{p}_{1}}
$$

which gives $C_{1}(t)=\frac{e^{2 b_{1}(t)}}{p_{1}+\bar{p}_{1}}$ in the small limit of $\varepsilon$. Furthermore, we can derive the following equations using (4.1-4.3) and (4.18):

$$
\begin{aligned}
& f=\frac{\left(1+2 a_{2} \beta_{1}\left(k_{2}\right)\right)^{\frac{1}{a_{2}}}}{p_{1}+\bar{p}_{1}}+\frac{1}{p_{1}+\bar{p}_{1}}\left(\frac{1+\bar{p}_{1} a_{1}}{1-p_{1} a_{1}}\right)^{\frac{k_{1}}{a_{1}}}\left(\frac{1+\bar{p}_{1} a_{2}}{1-p_{1} a_{2}}\right)^{\frac{k_{2}}{a_{2}}}\left(\frac{1+\bar{p}_{1} a_{3}}{1-p_{1} a_{3}}\right)^{\frac{k_{3}}{a_{3}}} \\
& =\frac{e^{2 \beta_{1}\left(k_{2}\right)+O(\varepsilon)}}{p_{1}+\bar{p}_{1}}+\frac{1}{p_{1}+\bar{p}_{1}} e^{\frac{k_{1}}{\delta} \ln \left(1+\bar{p}_{1} \delta\right)} e^{\frac{k_{2}}{\varepsilon} \ln \left(1+\bar{p}_{1} \varepsilon\right)} e^{k_{3} \ln \left(1+\bar{p}_{1}\right)} e^{-\frac{k_{1}}{\delta} \ln \left(1-p_{1} \delta\right)} e^{-\frac{k_{2}}{\varepsilon} \ln \left(1-p_{2} \varepsilon\right)} e^{-k_{3} \ln \left(1-p_{1}\right)} \\
& =\frac{1}{p_{1}+\bar{p}_{1}}\left(e^{2 b_{1}(t)+O(\varepsilon)}+e^{\left(p_{1}+\bar{p}_{1}\right) y+\left(\bar{p}_{1}^{2}-p_{1}^{2}\right) t+\left(\ln \left(1+\bar{p}_{1}\right)-\ln \left(1-p_{1}\right)\right) n+O(\delta)+O\left(\varepsilon^{2}\right)}\right), \\
& g=\frac{\left(1+2 a_{2} \beta_{1}\left(k_{2}\right)\right)^{\frac{1}{a_{2}}}}{p_{1}+\bar{p}_{1}}-\frac{\bar{p}_{1}}{p_{1}\left(p_{1}+\bar{p}_{1}\right)}\left(\frac{1+\bar{p}_{1} a_{1}}{1-p_{1} a_{1}}\right)^{\frac{k_{1}}{a_{1}}}\left(\frac{1+\bar{p}_{1} a_{2}}{1-p_{1} a_{2}}\right)^{\frac{k_{2}}{a_{2}}}\left(\frac{1+\bar{p}_{1} a_{3}}{1-p_{1} a_{3}}\right)^{\frac{k_{3}}{a_{3}}} \\
& =\frac{1}{p_{1}+\bar{p}_{1}}\left(e^{\left.2 b_{1}(t)\right)+O(\varepsilon)}-\frac{\bar{p}_{1}}{p_{1}} e^{\left(p_{1}+\bar{p}_{1}\right) y+\left(\bar{p}_{1}^{2}-p_{1}^{2}\right) t+\left(\ln \left(1+\bar{p}_{1}\right)-\ln \left(1-p_{1}\right)\right) n+O(\delta)+O\left(\varepsilon^{2}\right)}\right), \\
& P_{11}=\sqrt{c_{1}\left(k_{2}\right)-c_{1}\left(k_{2}-a_{2}\right)}\left(1+\bar{p}_{1} a_{1}\right)^{\frac{k_{1}}{a_{1}}}\left(1+\bar{p}_{1} a_{2}\right)^{\frac{k_{2}}{a_{2}}}\left(1+\bar{p}_{1} a_{3}\right)^{\frac{k_{3}}{a_{3}}} \\
& =\sqrt{\varepsilon \frac{d c_{1}\left(k_{2}\right)}{d k_{2}}+O\left(\varepsilon^{2}\right)} e^{\frac{k_{1}}{\delta} \ln \left(1+\bar{p}_{1} \delta\right)} e^{\frac{k_{2}}{\varepsilon} \ln \left(1+\bar{p}_{1} \varepsilon\right)} e^{k_{3} \ln \left(1+\bar{p}_{1}\right)} \\
& =\sqrt{-\frac{1}{2} \varepsilon^{2} \frac{d C_{1}(t)}{d t}+O\left(\varepsilon^{4}\right)} e^{\bar{p}_{1} y+\bar{p}_{1}^{2} t+\ln \left(1+\bar{p}_{1}\right) n+O(\delta)+O\left(\varepsilon^{2}\right)} \\
& =\varepsilon \sqrt{-\frac{1}{2} \frac{d C_{1}(t)}{d t}+O\left(\varepsilon^{2}\right)} e^{\bar{p}_{1} y+\bar{p}_{1}^{2} t+\ln \left(1+\bar{p}_{1}\right) n+O(\delta)+O\left(\varepsilon^{2}\right)},
\end{aligned}
$$




$$
\begin{aligned}
Q_{11} & =\frac{1}{p_{1}} \sqrt{c_{1}\left(k_{2}+a_{2}\right)-c_{1}\left(k_{2}\right)}\left(1-p_{1} a_{1}\right)^{-\frac{k_{1}}{a_{1}}}\left(1-p_{1} a_{2}\right)^{-\frac{k_{2}}{a_{2}}}\left(1-p_{1} a_{3}\right)^{-\frac{k_{3}}{a_{3}}} \\
& =\sqrt{\varepsilon \frac{d c_{1}\left(k_{2}\right)}{d k_{2}}+O\left(\varepsilon^{2}\right)} e^{-\frac{k_{1}}{\delta} \ln \left(1-p_{1} \delta\right)} e^{-\frac{k_{2}}{\varepsilon} \ln \left(1-p_{1} \varepsilon\right)} e^{-k_{3} \ln \left(1-p_{1}\right)} \\
& =\sqrt{-\frac{1}{2} \varepsilon^{2} \frac{d C_{1}(t)}{d t}+O\left(\varepsilon^{4}\right)} e^{p_{1} y-p_{1}^{2} t-\ln \left(1-p_{1}\right) n+O(\delta)+O\left(\varepsilon^{2}\right)} \\
& =\varepsilon \sqrt{-\frac{1}{2} \frac{d C_{1}(t)}{d t}+O\left(\varepsilon^{2}\right)} e^{p_{1} y-p_{1}^{2} t-\ln \left(1-p_{1}\right) n+O(\delta)+O\left(\varepsilon^{2}\right)} .
\end{aligned}
$$

From equations (4.3) and (4.24-4.25), we have

$$
\begin{aligned}
& H_{11}=\sqrt{-\frac{1}{2} \frac{d C_{1}(t)}{d t}+O\left(\varepsilon^{2}\right)} e^{\bar{p}_{1} y+\bar{p}_{1}^{2} t+\ln \left(1+\bar{p}_{1}\right) n+O(\delta)+O\left(\varepsilon^{2}\right)}, \\
& S_{11}=\sqrt{-\frac{1}{2} \frac{d C_{1}(t)}{d t}+O\left(\varepsilon^{2}\right)} e^{p_{1} y-p_{1}^{2} t-\ln \left(1-p_{1}\right) n+O(\delta)+O\left(\varepsilon^{2}\right)}
\end{aligned}
$$

Through the small limit of $\delta$ and $\varepsilon$, the functions $f, g, H_{11}, S_{11}$ given in (4.20-4.22,4.26-4.27) become

$$
\begin{aligned}
& f=\frac{e^{2 b_{1}(t)}}{p_{1}+\bar{p}_{1}}\left(1+e^{\left(p_{1}+\bar{p}_{1}\right) y+\left(\bar{p}_{1}^{2}-p_{1}^{2}\right) t+\left(\ln \left(1+\bar{p}_{1}\right)-\ln \left(1-p_{1}\right)\right) n-2 b_{1}(t)}\right), \\
& g=\frac{e^{2 b_{1}(t)}}{p_{1}+\bar{p}_{1}}\left(1-\frac{\bar{p}_{1}}{p_{1}} e^{\left(p_{1}+\bar{p}_{1}\right) y+\left(\bar{p}_{1}^{2}-p_{1}^{2}\right) t+\left(\ln \left(1+\bar{p}_{1}\right)-\ln \left(1-p_{1}\right)\right) n-2 b_{1}(t)}\right), \\
& H_{11}=\sqrt{-\frac{1}{2} \frac{d C_{1}(t)}{d t}} e^{\bar{p}_{1} y+\bar{p}_{1}^{2} t+\ln \left(1+\bar{p}_{1}\right) n}=\sqrt{-\frac{\dot{b}_{1}(t)}{p_{1}+\bar{p}_{1}}} e^{\bar{p}_{1} y+\bar{p}_{1}^{2} t+\ln \left(1+\bar{p}_{1}\right) n+b_{1}(t),}
\end{aligned}
$$

and

$$
S_{11}=\sqrt{-\frac{1}{2} \frac{d C_{1}(t)}{d t}} e^{p_{1} y-p_{1}^{2} t-\ln \left(1-p_{1}\right) n}=\sqrt{-\frac{\dot{b}_{1}(t)}{p_{1}+\bar{p}_{1}}} e^{p_{1} y-p_{1}^{2} t-\ln \left(1-p_{1}\right) n+b_{1}(t)},
$$

respectively. We can verify that the functions $f, g, H_{11}, S_{11}$ given in (4.28-4.31) solve the modified differential-difference KP equation with self-consistent sources (4.4-4.7).

\section{Acknowledgments}

One of the authors (Gegenhasi) would like to express her sincere thanks to Prof. Xing-Biao Hu for his helpful discussions and encouragement. The authors appreciate the valuable suggestions of the referees which improve the quality of the paper. This work was supported by the Natural Science Foundation of Inner Mongolia Autonomous Region (Grant no.2016MS0115), the National Natural Science Foundation of China (Grant no. 11601247 and 11605096) and the Innovation Foundation of Inner Mongolia University for the College Students (201511141). 


\section{References}

[1] R. Hirota, Discrete analogue of a generalized Toda equation, J. Phys. Soc. Japan 50 (1981) 3785-3791.

[2] T. Miwa, On Hirota's difference equation, Proc. Japan. Acad. A 58 (1982) 9-12.

[3] Y. Ohta, R.Hirota and S.Tsujimoto,T.Imai, Casorati and discrete Gram type determinant representations of solutions to the discrete KP hierarchy, J. Phys. Soc. Japan 62 (1993) 1872-1886.

[4] N. Shinzawa and R. Hirota, Bäcklund transformation equations for the ultradiscrete KP equation, J.Phys. A:Math. Gen. 36 (2003) 4667-4675.

[5] V. K. Mel'nikov, On equations for wave interactions, Lett. Math. Phys. 7 (1983) 129-136.

[6] V. K. Mel'nikov, Integration of the Korteweg-de Vries equation with a source, Inverse Problems 6 (1990) 233-246.

[7] V. K. Mel'nikov, Integration of the nonlinear Schrödinger equation with a source, Inverse Problems 8 (1992) 133-147.

[8] Y. B. Zeng, W .X. Ma, R. L. Lin, Integration of the soliton hierarchy with self-consistent sources, J. Math. Phys. 41 (2000) 5453-5489..

[9] R. L. Lin, Y. B. Zeng, W. X. Ma, Solving the KdV hierarchy with self-consistent sources by inverse scattering method, Physica A 291 (2001) 287-298.

[10] Y. B. Zeng, W.X.Ma, Y.J.Shao, Two binary Darboux transformations for the KdV hierarchy with selfconsistent sources, J. Math. Phys. 42 (2001) 2113-2128.

[11] Y. B. Zeng, Y. J. Shao, W. X. Ma, Integrable-type Darboux transformation for the $\mathrm{mKdV}$ hierarchy with self-consistent sources, Commun. Theor. Phys. (Beijing) 38 (2002) 641-648.

[12] T. Xiao, Y. B. Zeng, Generalized Darboux transformations for the KP equation with self-consistent sources, J. Phys. A: Math. Gen. 37 (2004) 7143-7162.

[13] X. J. Liu, Y. B. Zeng, On the Toda lattice equation with self-consistent sources, J. Phys. A: Math. Gen. 38 (2005) 8951-8965.

[14] Y. Hase, R. Hirota, Y. Ohta, J. Satsuma, Soliton solutions of the Mel'nikov equations, J. Phys. Soc. Jpn. 58 (1989) 2713-2720.

[15] Y. Matsuno, Bilinear Bäcklund transformation for the KdV equation with a source, J. Phys. A: Math. Gen. 24 (1991) L273-L277.

[16] X. B .Hu, Nonlinear superposition formula of the KdV equation with a source, J. Phys. A: Math. Gen. 24 (1991) 5489-5497.

[17] Y. Matsuno, KP equation with a source and its soliton solutions, J. Phys. A: Math. Gen. 23 (1990) L1235-L1239.

[18] D. J. Zhang, The N-soliton solutions for the modified KdV equation with self-consistent sources, $J$. Phys. Soc. Jpn. 71 (2002) 2649-2656.

[19] S. F.Deng, D. Y. Chen, D. J. Zhang, The multisoliton solutions of the KP equation with self-consistent sources, J. Phys.Soc. Jpn. 72 (2003) 2184-2192.

[20] D. J. Zhang, D. Y. Chen, The N-soliton solutions of the sine-Gordon equation with self- consistent sources, Physica A 321 (2003) 467-481.

[21] D. J. Zhang, The N-soliton solutions of some soliton equations with self-consistent sources, Chaos, Solitons and Fractals 18 (2003) 31-43.

[22] Gegenhasi, X. B. Hu, On a integrable differential-difference equation with a source, J. Nonlinear Math.Phys. 13 (2006) 183-192.

[23] X. B. Hu, H. Y. Wang, Construction of dKP and BKP equation with self-consistent sources, Inverse Problems 22 (2006) 1903-1920.

[24] Gegenhasi, X. B. Hu, H. W. Tam, A pfaffian version of the modified discrete KP equation, J. Math. Anal. Appl. 311 (2005) 518-529.

[25] J X Zhao, Gegenhasi, X B Hu, Commutativity of pfaffianization and Bäcklund transformations: two differential-difference systems, J. Phys. Soc. Jpn. 78 (2009) 064005. 